\title{
Renormalization group constraints on new top interactions from electroweak precision data
}

\author{
J. de Blas, ${ }^{a}$ M. Chala ${ }^{b}$ and J. Santiago ${ }^{c}$ \\ ${ }^{a}$ INFN - Sezione di Roma, \\ Piazzale A. Moro 2, I-00185 Rome, Italy \\ ${ }^{b} D E S Y$, \\ Notkestrasse 85, 22607 Hamburg, Germany \\ ${ }^{c}$ Departamento de Física Teórica y del Cosmos and CAFPE, \\ Universidad de Granada, Campus de Fuentenueva, E-18071 Granada, Spain \\ E-mail: Jorge.DeBlasMateo@roma1.infn.it, mikael.chala@desy.de, \\ jsantiago@ugr.es
}

ABSTRACT: Anomalous interactions involving the top quark contribute to some of the most difficult observables to directly access experimentally. They can give however a sizeable correction to very precisely measured observables at the loop level. Using a model-independent effective Lagrangian approach, we present the leading indirect constraints on dimension-six effective operators involving the top quark from electroweak precision data. They represent the most stringent constraints on these interactions, some of which may be directly testable in future colliders.

Keywords: Beyond Standard Model, Phenomenological Models, Renormalization Group

ARXIV EPRINT: 1507.00757 


\section{Contents}

1 Introduction 1

2 The global electroweak fit for new physics to dimension six 2

3 Loop constraints on new top interactions 4

$\begin{array}{llr}4 & \text { Conclusions } & 9\end{array}$

\section{Introduction}

Once the presence of the Higgs boson has been firmly established at the Large Hadron Collider (LHC) the focus has turned towards the discovery of new physics (NP). Already at Run 1 the absence of significant deviations from the Standard Model (SM) predictions has put stringent bounds on the mass scale of NP. Indirect constraints on new particles, when they are beyond the kinematic reach of the LHC, are becoming competitive and in some cases complementary to the indirect constraints from electroweak precision data (EWPD) [1]. It is expected that, with the increased energy available during Run 2, any new particle within the kinematic reach will be discovered, or very stringent constraints will be placed in case they cannot be directly produced. Still, there are certain interactions that, even with the increased energy, will be very difficult to directly probe at the LHC with a significant precision. A notable example is that of the interactions involving the top quark that we consider in this work.

Many well-motivated models of NP predict the largest deviations from the SM results in processes involving the top quark. Using an effective Lagrangian description, several groups have studied the potential of the LHC to constrain higher-dimensional operators involving the top quark in single and pair top production [2-11], including in some cases next-toleading order predictions (see [12-14] and references therein). Some of these interactions can be directly constrained to a reasonable accuracy for the first time at the LHC. However, the complexity of the $t(\bar{t})$ system limits the precision that one can achieve with these direct probes. In some cases, certain higher-dimensional operators are just inaccessible at the LHC. Many of these interactions, however, contribute at the loop level to EWPD. The very stringent constraints that can be derived from EWPD, together with the fact that the relevant coupling is usually the top Yukawa coupling, can compensate for the loop suppression, thus producing the most stringent constraints on many higher-dimensional operators involving the top quark. In particular, this includes those that cannot be directly probed at the LHC. 
In this article we use EWPD to place bounds on NP interactions. We use a modelindependent effective Lagrangian approach going beyond the usual analysis of dimensionsix operators correcting precision observables at the tree level. Analyses including oneloop contributions from higher-dimensional operators have been done for a small subset of purely bosonic operators in the past [15-19]. Here we use the calculation of the renormalization group equations (RGE) for the entire dimension-six effective Lagrangian [20-22] (see also [23-26]) to determine, without restricting to any particular set of operators, which interactions can be constrained by EWPD at the $O(0.1)$ or better. This precision can be achieved by higher-dimensional operators that give a sizeable loop contribution to EWPD. If we further neglect operators that can be directly probed in current or past experiments, we are basically left with dimension-six operators involving top quarks. We will show that indirect constraints from EWPD can be quite stringent for these interactions, some of which could be tested in future lepton colliders.

We discuss the global fit to EWPD, including the leading, currently unconstrained, loop effects in section 2. The corresponding constraints on the coefficients of the dimension-six operators involving the top quark are presented in section 3 and we conclude in section 4 .

\section{The global electroweak fit for new physics to dimension six}

Assuming that NP is heavier than the energies currently probed by experiments, its effects can be described by an effective Lagrangian,

$$
\mathcal{L}_{\mathrm{Eff}}=\mathcal{L}_{\mathrm{SM}}+\frac{1}{\Lambda^{2}} \mathcal{L}_{6}+\ldots
$$

where $\mathcal{L}_{d}=\sum c_{i} \mathcal{O}_{i}$, with $\mathcal{O}_{i}$ Lorentz and SM gauge invariant operators of mass dimension $d$ built from the SM fields. $\Lambda$ stands for the cut-off scale of the effective theory, and we have neglected lepton number violating effects. There is a total of 59 operators (up to flavor indices) at dimension six [27]. However, only a few of these directly contribute at leading order to EWPD. We consider in our analysis the following set of EWPD:

$$
\begin{aligned}
& \left\{M_{H}, m_{t}, \alpha_{S}\left(M_{Z}^{2}\right), \Delta \alpha_{\mathrm{had}}^{(5)}\left(M_{Z}^{2}\right), M_{W}, \Gamma_{W}, \mathrm{Br}_{W}^{e \nu, \mu \nu, \tau \nu}, M_{Z}, \Gamma_{Z}, \sigma_{\mathrm{had}}, R_{e, \mu, \tau}\right. \\
& \left.A_{F B}^{e, \mu, \tau}, A_{e, \mu, \tau}(\mathrm{SLD}), A_{e, \tau}\left(P_{\tau}\right), R_{b, c}, A_{\mathrm{FB}}^{b, c}, A_{b, c}, A_{\mathrm{FB}}^{s}, A_{s}, R_{u} / R_{u+d+s}, Q_{\mathrm{FB}}^{\mathrm{had}}\right\}
\end{aligned}
$$

whose definition, experimental values, errors and correlation matrices are taken from [28$34]$.

With these observables we perform a fit to the SM extended with the relevant set of dimension-six interactions that we will introduce below. The SM predictions for EWPD are computed including the latest theoretical developments [35-38]. These are then corrected by the NP dimension-six operators, and compared to the experimental measurements via the usual $\chi^{2}$ function, which we minimize and use to determine the bounds on the new interactions. In our fits both the NP and the SM parameters are allowed to float. In order to include dimension-six effects in a consistent way, we limit the contributions to EWPD to order $1 / \Lambda^{2}$. These may come either from the direct interference of the NP amplitudes 
with the SM ones in the electroweak precision observables, or from NP contributions to the physical processes where some of the SM input parameters are determined (e.g. $G_{F}$ as extracted from muon decay), which then propagate to all observables. Effects of order $1 / \Lambda^{4}$ would be comparable to interference effects coming from dimension-eight operators and have been therefore neglected. Given the resulting bounds, this seems in general a good approximation. An analysis of the error induced by neglecting such contributions, along the lines explained in e.g. [39], goes beyond the scope of this paper.

The analysis of bounds on dimension-six interactions from EWPD has been considered extensively in the literature (see for instance [40-46]). At the tree level, only the following coefficients of dimension-six operators modify the observables in (2.2):

$$
c_{\mathrm{EWPD}}^{\text {tree }}=\left\{c_{\phi l}^{(1)}, c_{\phi q}^{(1)}, c_{\phi e}^{(1)}, c_{\phi u}^{(1)}, c_{\phi d}^{(1)}, c_{\phi l}^{(3)}, c_{\phi q}^{(3)}, c_{\phi D}, c_{W B}, c_{l l}^{(1)}\right\} .
$$

The definition of the operator basis used in this work follows closely the original one in [27] except for the four-fermion sector for which we use the one in appendix A of [47]. ${ }^{1}$ At the loop level, however, many other dimension-six operators contribute to EWPD, including some to which we have little or no direct experimental access. In this latter case, the high precision of the EWPD can compensate the loop suppression and give the most stringent constraints on these operators. The complete one-loop calculation of the EWPD including dimension-six operators is beyond the scope of this article but the logarithmically enhanced contributions can be computed by means of the RGE recently computed in [20-22]. An analysis of the loop-improved electroweak constraints on the dimension-six interactions in (2.3) will be presented elsewhere [48]. Here we want to focus on those operators that, as explained above, have not been directly probed by any experiment yet, and to determine which ones can be constrained to a reasonable accuracy with current data.

The largest RGE effects on EWPD are those proportional to the top Yukawa coupling, $y_{t}$. (The strong coupling $g_{3}$ does not enter in any of the anomalous dimensions for the interactions in (2.3) .) Inspecting the RGE for the operators in (2.3) and looking for $y_{t}$ effects then allows us to identify which ones could be significantly constrained from the lowenergy bounds. Restricting to operators that have not been directly tested in experiments so far leaves us with the following set, containing always the top quark: ${ }^{2}$

$$
\begin{aligned}
& \mathcal{O}_{\phi q}^{(1)}=\left(\phi^{\dagger} i \stackrel{\leftrightarrow}{D_{\mu}} \phi\right)\left(\overline{q_{L}} \gamma^{\mu} q_{L}\right), \quad \mathcal{O}_{\phi q}^{(3)}=\left(\phi^{\dagger} i \stackrel{\leftrightarrow}{D_{\mu}^{a}} \phi\right)\left(\overline{q_{L}} \gamma^{\mu} \sigma_{a} q_{L}\right), \\
& \mathcal{O}_{\phi u}^{(1)}=\left(\phi^{\dagger} \stackrel{\leftrightarrow}{D_{\mu}} \phi\right)\left(\overline{u_{R}} \gamma^{\mu} u_{R}\right) \\
& \mathcal{O}_{l q}^{(1)}=\left(\overline{l_{L}} \gamma_{\mu} l_{L}\right)\left(\overline{q_{L}} \gamma^{\mu} q_{L}\right), \quad \mathcal{O}_{l q}^{(3)}=\left(\overline{l_{L}} \gamma_{\mu} \sigma_{a} l_{L}\right)\left(\overline{q_{L}} \gamma^{\mu} \sigma_{a} q_{L}\right),
\end{aligned}
$$

\footnotetext{
${ }^{1}$ For completeness, the dimension-six operators in (2.3) are presented here: $\mathcal{O}_{\phi \psi}^{(1)}=\left(\phi^{\dagger} i \stackrel{\leftrightarrow}{D_{\mu}} \phi\right)\left(\bar{\psi} \gamma^{\mu} \psi\right)$ $\mathcal{O}_{\phi \psi}^{(3)}=\left(\phi^{\dagger} i \stackrel{\leftrightarrow}{D_{\mu}^{a}} \phi\right)\left(\overline{\psi_{L}} \gamma^{\mu} \sigma_{a} \psi_{L}\right)\left(\right.$ with $\stackrel{\leftrightarrow}{D}_{\mu}=\vec{D}_{\mu}-\overleftarrow{D}_{\mu}$ and $\left.\stackrel{\leftrightarrow}{D}_{\mu}^{a}=\sigma_{a} \vec{D}_{\mu}-\overleftarrow{D}_{\mu} \sigma_{a}\right), \mathcal{O}_{\phi D}=\left(\phi^{\dagger} D_{\mu} \phi\right)\left(\left(D^{\mu} \phi\right)^{\dagger} \phi\right)$ $\mathcal{O}_{W B}=\left(\phi^{\dagger} \sigma_{a} \phi\right) W_{\mu \nu}^{a} B^{\mu \nu}$ and $\mathcal{O}_{l l}^{(1)}=\frac{1}{2}\left(\overline{l_{L}} \gamma_{\mu} l_{L}\right)\left(\overline{l_{L}} \gamma^{\mu} l_{L}\right)$.

${ }^{2}$ Apart from these, we will also comment on another operator (see footnote 3 ): the pure scalar interaction $\mathcal{O}_{\phi \square}=\left(\phi^{\dagger} \phi\right) \square\left(\phi^{\dagger} \phi\right)$, which does not contribute to EWPD proportionally to $y_{t}$, but enters in the anomalous dimension of $c_{\phi D}$ with a large coefficient $\sim 20 g_{1}^{2} / 3$ [22]. Although this operator can be in principle tested in Higgs physics, its constraints are still very weak [49].
} 


$$
\begin{array}{rlrl}
\mathcal{O}_{e u} & =\left(\overline{e_{R}} \gamma_{\mu} e_{R}\right)\left(\overline{u_{R}} \gamma^{\mu} u_{R}\right), & \mathcal{O}_{l u} & =\left(\overline{l_{L}} \gamma_{\mu} l_{L}\right)\left(\overline{u_{R}} \gamma^{\mu} u_{R}\right), \\
\mathcal{O}_{q e} & =\left(\overline{q_{L}} \gamma_{\mu} q_{L}\right)\left(\overline{e_{R}} \gamma^{\mu} e_{R}\right), & & \\
\mathcal{O}_{q q}^{(1)} & =\frac{1}{2}\left(\overline{q_{L}} \gamma_{\mu} q_{L}\right)\left(\overline{q_{L}} \gamma^{\mu} q_{L}\right), & \mathcal{O}_{q q}^{(8)} & =\frac{1}{2}\left(\overline{q_{L}} \gamma_{\mu} T_{A} q_{L}\right)\left(\overline{q_{L}} \gamma^{\mu} T_{A} q_{L}\right), \\
\mathcal{O}_{u u}^{(1)} & =\frac{1}{2}\left(\overline{u_{R}} \gamma_{\mu} u_{R}\right)\left(\overline{u_{R}} \gamma^{\mu} u_{R}\right), & \mathcal{O}_{u d}^{(1)} & =\left(\overline{u_{R}} \gamma_{\mu} u_{R}\right)\left(\overline{d_{R}} \gamma^{\mu} d_{R}\right), \\
\mathcal{O}_{q u}^{(1)} & =\left(\overline{q_{L}} \gamma_{\mu} q_{L}\right)\left(\overline{u_{R}} \gamma^{\mu} u_{R}\right), & \mathcal{O}_{q d}^{(1)} & =\left(\overline{q_{L}} \gamma_{\mu} q_{L}\right)\left(\overline{d_{R}} \gamma^{\mu} d_{R}\right), \\
\mathcal{O}_{u B} & =\left(\overline{q_{L}} \sigma^{\mu \nu} u_{R}\right) \tilde{\phi} B_{\mu \nu}, & \mathcal{O}_{u W}=\left(\overline{q_{L}} \sigma^{\mu \nu} \sigma_{a} u_{R}\right) \tilde{\phi} W_{\mu \nu}^{a} .
\end{array}
$$

An analysis of EWPD constraints on a small subset of these operators was performed in [50]. However, at one loop only non-logarithmic finite contributions were included and the corresponding bounds are much weaker. Regarding the operators $\mathcal{O}_{\phi q}^{(1),(3)}$, we will only consider the combination $\mathcal{O}_{\phi q}^{(t)} \equiv\left(\mathcal{O}_{\phi q}^{(1)}-\mathcal{O}_{\phi q}^{(3)}\right)_{t t}$, which (up to corrections suppressed by products of $V_{t d}$ and $V_{t s}$ with $V$ the Cabibbo-Kobayashi-Maskawa matrix) modifies the neutral current top couplings, without inducing any tree-level correction in the bottom ones. As we will also see, because EWPD is only sensitive to $\mathcal{O}_{\phi q}^{(b)} \equiv\left(\mathcal{O}_{\phi q}^{(1)}+\mathcal{O}_{\phi q}^{(3)}\right)_{b b}$ at the tree level, NP effects coming from $\mathcal{O}_{q q}^{(8)}$ cannot be constrained by the data. Similarly, EWPD is not sensitive to $\left(\mathcal{O}_{\phi u}^{(1)}\right)_{t t}$, so $\mathcal{O}_{u u}^{(1)}$ cannot be constrained if it only involves the third generation.

As we mentioned above, we compute the predictions for physical observables consistently with the approximation of a dimension-six effective Lagrangian, including only the interference between the SM amplitudes and the NP effects (i.e. terms linear in $1 / \Lambda^{2}$ ). We also use the leading logarithmic approximation for the solution of the RGE

$$
\frac{d C_{i}}{d \log \mu}=\frac{1}{16 \pi^{2}} \gamma_{i}^{j} C_{j} \Longrightarrow C_{i}(\mu) \approx\left(\delta_{i}^{j}+\frac{1}{16 \pi^{2}} \gamma_{i}^{j}(\Lambda) \log \frac{\mu}{\Lambda}\right) C_{j}(\Lambda)
$$

where we have defined the dimension-six coefficients $C_{i} \equiv c_{i} / \Lambda^{2}$. We include in the anomalous dimensions $\gamma_{i}^{j}$ the full dependence on the SM gauge couplings [22] and the leading contributions from the Yukawa interactions [21], i.e. $Y_{e}, Y_{d} \approx 0$, and $Y_{u} \approx \operatorname{diag}\left(0,0, y_{t}\right)$. The dependence on the Higgs self-coupling [20] is irrelevant for our analysis. Note that, for the contributions to the EWPD that only arise from the RGE, within the approximation in eq. (2.5) the physical predictions depend always on $C_{i} \log \mu / \Lambda$. Finite one-loop contributions beyond the logarithmically enhanced terms included in the RGE can in some cases be relevant $[51,52]$, and therefore modify the previous dependence. Whenever these finite terms are available, we include them in our analysis. We explicitly comment on these cases below.

\section{Loop constraints on new top interactions}

In this section we present the constraints that EWPD impose on the operators involving the top quark in (2.4) due to RGE effects. We report these bounds in table 1 assuming 
that only one operator is generated at the ultraviolet scale, $\Lambda$, at a time. ${ }^{3}$ We present the results from two different types of fits. The limits on $C_{i} \log M_{Z} / \Lambda$ (and $c_{i}$, for $\Lambda=1 \mathrm{TeV}$ ) are obtained assuming that the dimension-six coefficients can have either sign. On the other hand, the bounds on the NP scale $\Lambda$ are derived from a fit with the extra assumption that the $c_{i}$ have a definite sign, and then setting this to some illustrative values, $c_{i}= \pm 1$. As we mentioned above, the precision of EWPD overcomes the loop suppression of the RGE effects and allows to constrain most of the interactions at the few percent level for $\Lambda=1 \mathrm{TeV}$, or alternatively pushes the scale of $\mathrm{NP}$ in the top sector to a few $\mathrm{TeV}$ for $c_{i}= \pm 1$, thus fully justifying the use of an effective Lagrangian description. (Note that, even for the weakest bounds, the NP scale is always pushed significantly above the $Z$ mass, where the EWPD are measured.) The leading-log approximation used is also justified provided the value of $\Lambda$ is not too large, so that $\left|\frac{\alpha_{i}}{4 \pi} \log \frac{M_{Z}}{\Lambda}\right| \ll 1$, with $\alpha_{i}$ the relevant SM parameter. In the rest of this section we discuss the origin of the constraints on the different operators.

The relatively strong constraints on $\ell^{+} \ell^{-} t \bar{t}$ interactions can be understood from the fact that all those interactions contribute to the running of $c_{\phi l}^{(1)}, c_{\phi l}^{(3)}$ or $c_{\phi e}^{(1)}$. The corresponding operators provide direct corrections to the neutral current couplings of the charged leptons, and are bounded at the per mile level (see [53] for an earlier partial analysis). Note that the bounds for some of these interactions, e.g. $\mathcal{O}_{l q}^{(1)}$ and $\mathcal{O}_{l u}$, are almost identical (up to a sign). This correlation follows directly from the RGE for the leptonic interactions in (2.3). In particular,

$$
\begin{aligned}
& \frac{d\left(C_{\phi l}^{(1)}\right)_{i j}}{d \log \mu}=\frac{N_{c}}{8 \pi^{2}}\left\{\left(Y_{u}^{\dagger} Y_{u}\right)_{l k}\left(C_{l q}^{(1)}\right)_{i j k l}-\left(Y_{u} Y_{u}^{\dagger}\right)_{l k}\left(C_{l u}\right)_{i j k l}\right\}+\ldots \\
& \frac{d\left(C_{\phi e}^{(1)}\right)_{i j}}{d \log \mu}=\frac{N_{c}}{8 \pi^{2}}\left\{\left(Y_{u}^{\dagger} Y_{u}\right)_{l k}\left(C_{q e}\right)_{k l i j}-\left(Y_{u} Y_{u}^{\dagger}\right)_{l k}\left(C_{e u}\right)_{i j k l}\right\}+\ldots
\end{aligned}
$$

Thus, only the combinations of operators appearing in eq. (3.2) can be constrained by EWPD, up to corrections in the RGE induced by the gauge interactions.

Constraints on four-quark interactions involving only the third family are dominated by the contributions they generate to the $Z b \bar{b}$ couplings, via the operators $\mathcal{O}_{\phi q}^{(1),(3)}$ and $\mathcal{O}_{\phi d}^{(1)}$, and are therefore somewhat weaker than the leptonic ones. Limits on $c_{q q}^{(1)}$ and $c_{q u}^{(1)}$ arise from the bounds on the left-handed bottom couplings, and are significantly stronger than those of $c_{u d}^{(1)}$ and $c_{q d}^{(1)}$, which contribute to the $Z b_{R} \overline{b_{R}}$ interactions. In particular, the strong preference for a positive (negative) value of $\left(c_{u d}^{(1)}\right)_{t t b b}\left(\left(c_{q d}^{(1)}\right)_{t t b b}\right)$ follows from the corresponding preference for a large correction to the right-handed bottom coupling, $\delta g_{R}^{b}=-\frac{1}{2}\left(c_{\phi d}^{(1)}\right)_{b b} \frac{v^{2}}{\Lambda^{2}}$, with $v \approx 246 \mathrm{GeV}$, to alleviate the $-2.5-\sigma$ deviation in the bottom forward-backward asymmetry at the $Z$-pole. Again, some of the bounds on these four-quark

\footnotetext{
${ }^{3}$ For completeness, we include here the $95 \%$ probability interval for the operator coefficient $c_{\phi \square}$ :

$$
\frac{c_{\phi \square}}{\Lambda^{2}} \log \frac{M_{Z}}{\Lambda} \in[-4.63,0.65] \mathrm{TeV}^{-2} \quad\left(c_{\phi \square} \in[-0.27,1.93] \text { for } \Lambda=1 \mathrm{TeV}\right) .
$$

For negative values of the coefficients, the corresponding bound is then somewhat better than the one obtained from Higgs observables [49].
} 


\begin{tabular}{|c|c|c|c|c|}
\hline \multirow[t]{2}{*}{ Operator } & $\begin{array}{l}\mathbf{9 5} \% \text { prc } \\
\frac{c_{i}}{\Lambda^{2}} \log \frac{M_{Z}}{\Lambda}\end{array}$ & $\begin{array}{c}\text { interval } \\
c_{i}\end{array}$ & \multicolumn{2}{|c|}{$\begin{array}{c}\text { 95\% prob. lower bound } \\
\Lambda[\mathrm{TeV}]\end{array}$} \\
\hline & {$\left[\mathbf{T e V}^{-2}\right]$} & $(\Lambda=1 \mathrm{TeV})$ & $\left(c_{i}=+1\right)$ & $\left(c_{i}=-1\right)$ \\
\hline$\left(\mathcal{O}_{l q}^{(1)}\right)_{e e t t}$ & {$[-0.15,0.38]$} & {$[-0.16,0.06]$} & 4.4 & 3.2 \\
\hline$\left(\mathcal{O}_{l q}^{(3)}\right)_{e e t t}$ & {$[-0.26,0.36]$} & {$[-0.15,0.11]$} & 3.7 & 3.3 \\
\hline$\left(\mathcal{O}_{\text {eu }}\right)_{\text {eett }}$ & {$[-0.21,0.44]$} & {$[-0.18,0.09]$} & 3.8 & 2.9 \\
\hline$\left(\mathcal{O}_{\text {lu }}\right)_{\text {eet }}$ & {$[-0.40,0.16]$} & {$[-0.07,0.17]$} & 3.1 & 4.3 \\
\hline$\left(\mathcal{O}_{q e}\right)_{t t e e}$ & {$[-0.42,0.20]$} & {$[-0.08,0.18]$} & 3 & 3.9 \\
\hline$\left(\mathcal{O}_{l q}^{(1)}\right)_{\mu \mu t t}$ & {$[-0.91,0.25]$} & {$[-0.11,0.38]$} & 1.9 & 2.9 \\
\hline$\left(\mathcal{O}_{l q}^{(3)}\right)_{\mu \mu t t}$ & {$[-0.04,0.54]$} & {$[-0.22,0.02]$} & 4.8 & 2.6 \\
\hline$\left(\mathcal{O}_{e u}\right)_{\mu \mu t t}$ & {$[-1.29,0.22]$} & {$[-0.09,0.54]$} & 1.5 & 2.6 \\
\hline$\left(\mathcal{O}_{l u}\right)_{\mu \mu t t}$ & {$[-0.26,0.95]$} & {$[-0.40,0.11]$} & 2.8 & 1.9 \\
\hline$\left(\mathcal{O}_{q e}\right)_{t t \mu \mu}$ & {$[-0.22,1.24]$} & {$[-0.52,0.09]$} & 2.7 & 1.6 \\
\hline$\left(\mathcal{O}_{l q}^{(1)}\right)_{\tau \tau t t}$ & {$[-0.52,0.96]$} & {$[-0.40,0.22]$} & 2.3 & 1.8 \\
\hline$\left(\mathcal{O}_{l q}^{(3)}\right)_{\tau \tau t t}$ & {$[-0.86,0.69]$} & {$[-0.29,0.36]$} & 1.9 & 2.1 \\
\hline$\left(\mathcal{O}_{e u}\right)_{\tau \tau t t}$ & {$[-0.58,1.18]$} & {$[-0.49,0.24]$} & 2.1 & 1.6 \\
\hline$\left(\mathcal{O}_{l u}\right)_{\tau \tau t t}$ & {$[-1.01,0.54]$} & {$[-0.23,0.42]$} & 1.8 & 2.2 \\
\hline$\left(\mathcal{O}_{q e}\right)_{t t \tau \tau}$ & {$[-1.14,0.56]$} & {$[-0.23,0.48]$} & 1.7 & 2.2 \\
\hline$\left(\mathcal{O}_{l q}^{(1)}\right)_{\ell \ell t t}$ & {$[-0.16,0.26]$} & {$[-0.11,0.07]$} & 4.7 & 3.9 \\
\hline$\left(\mathcal{O}_{l q}^{(3)}\right)_{\ell \ell t t}$ & {$[-0.07,0.29]$} & {$[-0.12,0.03]$} & 5.9 & 3.8 \\
\hline$\left(\mathcal{O}_{e u}\right)_{\ell \ell t t}$ & {$[-0.24,0.33]$} & {$[-0.14,0.10]$} & 3.8 & 3.4 \\
\hline$\left(\mathcal{O}_{l u}\right)_{\ell \ell t t}$ & {$[-0.27,0.17]$} & {$[-0.07,0.11]$} & 3.8 & 4.6 \\
\hline$\left(\mathcal{O}_{q e}\right)_{t t \ell \ell}$ & {$[-0.32,0.23]$} & {$[-0.10,0.13]$} & 3.4 & 3.9 \\
\hline$\left(\mathcal{O}_{q q}^{(1)}\right)_{t t t t}$ & {$[-0.55,1.38]$} & {$[-0.58,0.23]$} & 2.1 & 1.5 \\
\hline$\left(\mathcal{O}_{u d}^{(1)}\right)_{t t b b}$ & {$[0.25,10.9]$} & {$[-4.6,-0.10]$} & 0.89 & 0.37 \\
\hline$\left(\mathcal{O}_{q u}^{(1)}\right)_{t t t t}$ & {$[-1.47,0.59]$} & {$[-0.25,0.62]$} & 1.4 & 2 \\
\hline$\left(\mathcal{O}_{q d}^{(1)}\right)_{t t b b}$ & {$[-9.7,-0.07]$} & {$[0.03,4.06]$} & 0.41 & 0.95 \\
\hline$\left(\mathcal{O}_{u B}\right)_{t t}$ & {$[-0.35,0.10]$} & {$[-0.04,0.15]$} & 3.4 & 5.1 \\
\hline$\left(\mathcal{O}_{u W}\right)_{t t}$ & {$[-0.39,0.11]$} & {$[-0.05,0.17]$} & 3.2 & 4.7 \\
\hline
\end{tabular}

Table 1. EWPD bounds on top interactions, assuming one operator at a time at the scale $\Lambda$. The bounds on the NP scale $\Lambda$ are obtained from two independent types of fits, assuming a definite sign for the coefficients $c_{i}$. The results for the operators $\left(\mathcal{O}_{i}\right)_{\ell \ell t t, t t \ell \ell}$ are obtained assuming lepton universality in the interactions. The bounds for $\left(\mathcal{O}_{q q}^{(8)}\right)_{t t t t}$ are too weak and have been omitted, while the operator coefficient for $\left(\mathcal{O}_{u u}^{(1)}\right)_{t t t t}$ cannot be constrained within our approximations (see text for details). 
operators can be easily correlated from the corresponding contributions to the running for the quark interactions in (2.3),

$$
\begin{gathered}
\frac{d\left(C_{\phi q}^{(1)}+C_{\phi q}^{(3)}\right)_{i j}}{d \log \mu}=\frac{N_{c}}{16 \pi^{2}}\left\{\left(Y_{u}^{\dagger} Y_{u}\right)_{l k}\left(\left(C_{q q}^{(1)}\right)_{i j k l}+\left(C_{q q}^{(1)}\right)_{k l i j}\right)\right. \\
\left.-2\left(Y_{u} Y_{u}^{\dagger}\right)_{l k}\left(C_{q u}^{(1)}\right)_{i j k l}\right\}+\ldots \\
\frac{d\left(C_{\phi d}^{(1)}\right)_{i j}}{d \log \mu}=\frac{N_{c}}{8 \pi^{2}}\left(Y_{u}^{\dagger} Y_{u}\right)_{l k}\left(\left(C_{q d}^{(1)}\right)_{k l i j}-\left(C_{u d}^{(1)}\right)_{k l i j}\right)+\ldots
\end{gathered}
$$

that determine which combinations of operators can be constrained by EWPD. In the first equation of eq. (3.3) there is no contribution from $\mathcal{O}_{q q}^{(8)}$, because the corresponding corrections to the running of $C_{\phi q}^{(1)}$ and $C_{\phi q}^{(3)}$ cancel each other. There is a suppressed contribution to the running of $C_{\phi q}^{(1)}+C_{\phi q}^{(3)}$ from $C_{q q}^{(8)}$ proportional to the electroweak gauge couplings, which results in much weaker constraints. This explains the absence of a bound on $C_{q q}^{(8)}$ in table 1. Finally, the coefficient $\left(C_{u u}^{(1)}\right)_{t t t t}$ only contributes to the $Z t_{R} \overline{t_{R}}$ couplings through the RGE for $\left(C_{\phi u}^{(1)}\right)_{t t}$, and therefore cannot be bounded by EWPD at the order we are working.

Four-quark operators involving two quarks of the third generation and two of either the first or second generations contribute, through RGE, to operators that modify the electroweak couplings of the quarks in the first two generations. These have been measured with worse precision than those of the charged leptons or bottom quark. Hence, the corresponding bounds are much weaker and not reported here. If one assumes universality among the three families then the bounds are still mostly dominated by the operators involving only third generation quarks. The exception is the case of the operators $\mathcal{O}_{q d, u d}^{(1)}$, for which there is a tension between the required contribution to $\delta g_{R}^{b}$ and the corresponding one for the first two generations. This tension results in significantly improved bounds, reducing the size of the corresponding $95 \%$ probability intervals by a factor of two, e.g. $\left(C_{q d}^{(1)}\right)_{t t q q} \log \frac{M_{Z}}{\Lambda} \in[-4.09,0.65] \mathrm{TeV}^{-2}$.

The limits on the electroweak top dipole interactions, $\left(\mathcal{O}_{u B}\right)_{t t}$ and $\left(\mathcal{O}_{u W}\right)_{t t}$, come exclusively from their contributions to the running of $c_{W B}\left(c_{W B} / \Lambda^{2} \in[-0.009,0.003] \mathrm{TeV}^{-2}\right.$ at $95 \%$ probability), which is related to the $S$ parameter [54]. Hence, only the approximate combination $g_{2}\left(C_{u B}\right)_{t t}+2 g_{1}\left(\frac{1}{6}+\frac{2}{3}\right)\left(C_{u W}\right)_{t t}$ (where the $1 / 6$ and $2 / 3$ factors are the $q_{L}$ and $u_{R}$ hypercharges, respectively), which enters in the RGE for $c_{W B}$, can be constrained.

Finally, we have not included in table 1 the constraints on the operators that induce direct corrections to the top electroweak couplings,

$$
\delta g_{L}^{t}=-\frac{1}{2}\left(V\left(c_{\phi q}^{(1)}-c_{\phi q}^{(3)}\right) V^{\dagger}\right)_{t t} \frac{v^{2}}{\Lambda^{2}}=-c_{\phi q}^{(t)} \frac{v^{2}}{\Lambda^{2}}, \quad \delta g_{R}^{t}=-\frac{1}{2}\left(c_{\phi u}^{(1)}\right)_{t t} \frac{v^{2}}{\Lambda^{2}} .
$$

Note that, to dimension six, the effects on the left-handed sector are also correlated with the direct corrections of the charged current couplings, $\delta V_{t b}=\left(V c_{\phi q}^{(3)}\right)_{t b} v^{2} / \Lambda^{2}$. (We work in a flavor basis in which the SM Yukawa couplings for the down sector are diagonal.) The constraints on the combinations in eq. (3.4) follow from the one-loop contributions to the 
$T$ parameter and corrections to the $Z b \bar{b}$ vertices. These corrections contain logarithmically enhanced terms that can be read from the RGE of the operators $\mathcal{O}_{\phi D}$ (equivalent to the $T$ parameter in our basis), $\mathcal{O}_{\phi q}^{(b)} \equiv\left(\mathcal{O}_{\phi q}^{(1)}+\mathcal{O}_{\phi q}^{(3)}\right)_{b b}$ and $\left(\mathcal{O}_{\phi d}^{(1)}\right)_{b b}$. We have also included finite (not proportional to logarithms) one-loop effects by integrating out the top quark with the anomalous couplings defined in eq. (3.4) $[55,56]$. In particular, the finite contribution to the $T$ parameter is given by

$$
\alpha \Delta T=\frac{N_{c}}{16 \pi^{2}} y_{t}^{2} \operatorname{Re}\left\{\left(V \alpha_{\phi q}^{(3)}\right)_{t b} V_{t b}^{*}\right\} \frac{v^{2}}{\Lambda^{2}} .
$$

Because of these finite terms, the $\chi^{2}$ depends on both $C_{i}$ and $C_{i} \log \frac{M_{Z}}{\Lambda}$, so we vary $C_{i}$ and $\Lambda$ independently in our fits. We impose $\Lambda \geq 1 \mathrm{TeV}$, to avoid regions where the effective Lagrangian description may break down. In the bounds below, when no mention to $\Lambda$ is made, we take the most conservative bound that is reached for $\Lambda=1 \mathrm{TeV}$.

Considering only one of the combinations in eq. (3.4) at a time we obtain the following $95 \%$ probability interval for $\delta g_{L}^{t} / g_{L}^{t} \mathrm{SM}$,

$$
\frac{\delta g_{L}^{t}}{g_{L}^{t} \mathrm{SM}} \in[-0.016,0.002] \quad\left(c_{\phi q}^{(t)} \in[-0.01,0.09]\right),
$$

while for $\delta g_{R}^{t} / g_{R}^{t}$ SM we get

$$
\frac{\delta g_{R}^{t}}{g_{R}^{t \text { SM }}} \in[-0.017,0.002] \quad\left(\left(c_{\phi u}^{(1)}\right)_{t t} \in[-0.08,0.01]\right),
$$

where $g_{L}^{t \text { SM }}=\frac{1}{2}-\frac{2}{3} \sin ^{2} \theta_{W}$ and $g_{R}^{t \text { SM }}=-\frac{2}{3} \sin ^{2} \theta_{W}$, with $\theta_{W}$ the weak angle. When we consider dimension-six effects correcting both electroweak top couplings at the same time, the $95 \%$ probability bounds change to:

$$
\begin{aligned}
\frac{\delta g_{L}^{t}}{g_{L}^{t} \mathrm{SM}} \in[-0.048,0.089], & \frac{\delta g_{R}^{t}}{g_{R}^{t \mathrm{SM}}} \in[-0.102,0.044] . \\
\left(c_{\phi q}^{(t)} \in[-0.52,0.28],\right. & \left.\left(c_{\phi u}^{(1)}\right)_{t t} \in[-0.50,0.21] .\right)
\end{aligned}
$$

The weaker bounds compared with eqs. (3.6) and (3.7) follow from a strong correlation $(\approx$ $-99 \%$ ) between these two couplings, as can be seen in figure 1 left, which can be understood from the leading logarithmic contributions. Indeed, neglecting the finite contributions, there is a large correlation between the corresponding dimension-six operators, which comes from the RGE for $C_{\phi D}$ (whose limit dominates the constraints in eqs. (3.6) and (3.7) via the $T$ parameter),

$$
\frac{d C_{\phi D}}{d \log \mu}=\frac{N_{c}}{2 \pi^{2}}\left\{\left(C_{\phi q}^{(1)}\right)_{i j}\left(Y_{u}^{\dagger} Y_{u}\right)_{j i}-\left(Y_{u}^{\dagger} Y_{u}\right)_{i j}\left(C_{\phi u}^{(1)}\right)_{j i}\right\}+\ldots
$$

At this level there is an approximate flat direction for $\delta g_{L}^{t} / g_{L}^{t \text { SM }}=-\delta g_{R}^{t} / g_{R}^{t \text { SM }}$ (we have used $g_{L}^{t \text { SM }} \approx-2 g_{R}^{t \text { SM }}$ and $\left.c_{\phi q}^{(3)}=-c_{\phi q}^{(1)}\right)$, which is also reflected in the equality of the bounds in eqs. (3.6) and (3.7). This flat direction is lifted however by the logarithmic contributions to the $Z b_{L} \overline{b_{L}}$ vertex and, to a less extent, the contributions in the running 

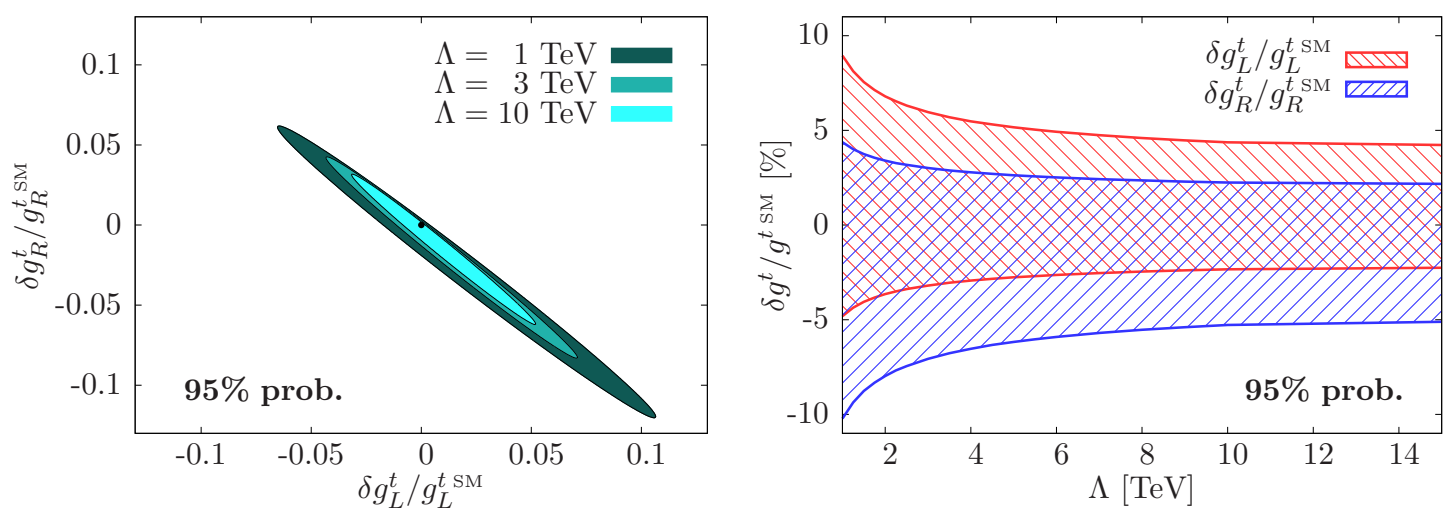

Figure 1. (Left) $95 \%$ probability regions in the $\frac{\delta g_{L}^{t}}{g_{L}^{t} \mathrm{SM}}-\frac{\delta g_{R}^{t}}{g_{R}^{t} \mathrm{SM}}$ plane for $\Lambda=1,3$ and $10 \mathrm{TeV}$. (Right) Boundaries of the $95 \%$ probability intervals for $\frac{\delta g_{L, R}^{t}}{g_{L, R}^{t} \text { SM }}$ as a function of $\Lambda$.

from gauge interactions. The global factors in front of the finite terms turn out to be smaller than the ones in the logarithmic terms, so the effects from the former are not very important for values of $\Lambda$ consistent with the effective Lagrangian description. In figure 1 right, we show how the bounds obtained in eq. (3.8) for $\Lambda=1 \mathrm{TeV}$ evolve as we increase the value of the scale of NP.

The results in eq. (3.8) imply quite strong bounds on deviations with the respect to the SM electroweak theory, at the few percent level. One may wonder though about the robustness of these bounds when interpreted within particular models. For instance, as explained above, the effects in the $T$ parameter have a strong impact in these bounds. Now, the $T$ parameter is known to have a strong correlation with the $S$ parameter (we obtain an $89 \%$ correlation), and many SM extensions in which the NP interacts mainly with the third generation come also usually accompanied by relatively large positive contributions to $S$. Still, considering a positive value for $S=0.2$ (about the $95 \%$ probability limit obtained from the $S-T$ fit) does not have a dramatic impact on the bounds in eq. (3.8), due to the constraints on the logarithmic contributions to $Z b_{L} \overline{b_{L}}$. We thus obtain only a moderate shift in the bounds for the right-handed couplings,

$$
\frac{\delta g_{L}^{t}}{g_{L}^{t \mathrm{SM}}} \in[-0.050,0.088], \quad \frac{\delta g_{R}^{t}}{g_{R}^{t \mathrm{SM}}} \in[-0.123,0.023] \quad(S=0.2),
$$

but we can still conclude that NP contributions to $Z t \bar{t}$ couplings beyond $10 \%$ are disfavored by the data.

\section{Conclusions}

Among the possible NP deformations of the SM there are several on which no direct experimental information can be extracted from present or past experiments. Indirect constraints can however be obtained in some cases from their loop contribution to the very precisely measured EWPD. Using a model-independent effective Lagrangian approach, we 
have explored in this article the potential that EWPD have to put constraints on dimensionsix effective operators on which no precise direct information is currently available. We have shown that, despite the loop suppression, the leading contributions proportional to the top Yukawa coupling are large enough to place significant bounds on several interactions involving the top quark.

Our results, reported in table 1, show that, for NP in the TeV region, EWPD can constrain the dimension-six operator coefficients for a large set of interactions involving the top quark to $O(0.1)$ values. These results are obtained using the logarithmically enhanced one-loop contributions, as given by the RGE, together with the finite terms in the most significant cases. Barring accidental cancellations, similar bounds will apply for all the interactions we have considered, even if the missing finite terms give a comparable effect to the logarithmically enhanced ones.

The bounds presented here have been computed assuming only one operator at a time at the scale of NP. There are several scenarios with new scalars, quarks or vector bosons for which such operators can be generated alone, or whose effects are not correlated with the contributions to other dimension-six interactions [47, 57, 58]. Nevertheless, we have discussed the origin of the leading constraints and described the most relevant approximate flat directions so that bounds on more complicated models can be estimated. As an example, let us discuss the case of lepton-top four-fermion interactions. For NP at the TeV scale we are able to place $O(0.1)$ bounds on the coefficients of contact interactions resulting from the products of vector currents involving two electron and two top fields, with all possible chiralities. As we have mentioned, the effects of some of these operators are correlated in the RGE that enter in the EWPD so, in practice, we can only constrain three combinations of these $e^{+} e^{-} t \bar{t}$ operators. Removing the two redundant interactions from a simultaneous global fit to all the operators we obtain the following limits (for $\Lambda=1 \mathrm{TeV}$ ):

$$
\begin{aligned}
\left(c_{l q}^{(1)}-c_{l u}\right)_{\text {eett }} & \in[-0.21,0.08], \\
\left(c_{l q}^{(3)}\right)_{\text {eett }} & \in[-0.31,0.06], \\
\left(c_{q e}-c_{\text {eu }}\right)_{\text {eett }} & \in[-0.10,0.29] .
\end{aligned}
$$

In particular, these $e^{+} e^{-} t \bar{t}$ contact interactions can be tested in future colliders [59], like the ILC [60] or the FCC-ee [61], and are therefore relevant to guide NP searches in these facilities. The same applies for our analysis of the $Z t \bar{t}$ couplings, which could be directly measured at this kind of experiments with great precision (around $1 \%$ for the ILC with $\left.500 \mathrm{fb}^{-1}[62]\right)$. Finally, while less precise, our limits on four-top interactions are comparable (and in some cases significantly better) than the latest ones obtained by the LHC [63].

Higher-dimensional operators involving the top quark are not only among the least constrained operators that contribute the most to EWPD, they are also among the best motivated ones in models that attempt to solve the hierarchy problem. In many of these models, a sizeable contribution to the $S$ parameter is generated at the NP scale. We have considered that possibility, see eq. (3.10), and shown that while inducing a shift in the allowed values of the coefficients of the higher-dimensional operators involving the top, these can still be constrained to a similar level of accuracy. This also illustrates the fact 
that, being indirect bounds, the limits we have computed are sensitive to assumptions about operators that can give tree-level contribution to EWPD. In the presence of such operators the quantitative results may change but the qualitative fact that one-loop contributions can place the most stringent bounds on currently untested operators still holds. These bounds are therefore a crucial piece of information, both for model building purposes and as a guide for future experimental searches.

Note added: while this manuscript was being prepared for submission, ref. [64] appeared in the arXiv. In that work, a global fit to dimension-six effective operators involving the top quark is performed using top production data from the LHC and the Tevatron. Our approach is complementary to theirs, in the sense that our analysis is sensitive to a different class of operators. In the few cases in which there is overlap, our results provide more stringent bounds on the coefficients of the operators.

\section{Acknowledgments}

We thank L. Silvestrini for useful discussions. The work of JB has been supported by the European Research Council under the European Union's Seventh Framework Programme (FP/2007-2013)/ERC Grant Agreement n. 279972. The work of JS has been partially supported by the European Commission (PITN-GA-2012-316704 HIGGSTOOLS), by MINECO under grants (FPA2010-17915 and FPA2013-47836-C3-2-P) and by Junta de Andalucía grants FQM 101 and FQM 6552. JS thanks the Pauli Center Visitor Program for financial support.

Open Access. This article is distributed under the terms of the Creative Commons Attribution License (CC-BY 4.0), which permits any use, distribution and reproduction in any medium, provided the original author(s) and source are credited.

\section{References}

[1] J. de Blas, M. Chala and J. Santiago, Global constraints on lepton-quark contact interactions, Phys. Rev. D 88 (2013) 095011 [arXiv: 1307.5068] [InSPIRE].

[2] J.A. Aguilar-Saavedra, A minimal set of top-Higgs anomalous couplings, Nucl. Phys. B 821 (2009) 215 [arXiv:0904.2387] [InSPIRE

[3] J.A. Aguilar-Saavedra, Effective four-fermion operators in top physics: a roadmap, Nucl. Phys. B 843 (2011) 638 [Erratum ibid. B 851 (2011) 443] [arXiv:1008.3562] [INSPIRE].

[4] C. Zhang and S. Willenbrock, Effective-field-theory approach to top-quark production and decay, Phys. Rev. D 83 (2011) 034006 [arXiv: 1008.3869] [inSPIRE].

[5] C. Degrande, J.-M. Gerard, C. Grojean, F. Maltoni and G. Servant, Non-resonant new physics in top pair production at hadron colliders, JHEP 03 (2011) 125 [arXiv:1010.6304] [INSPIRE].

[6] J.A. Aguilar-Saavedra and M. Pérez-Victoria, Probing the tevatron $t \bar{t}$ asymmetry at LHC, JHEP 05 (2011) 034 [arXiv:1103.2765] [INSPIRE]. 
[7] C. Degrande, J.-M. Gerard, C. Grojean, F. Maltoni and G. Servant, An effective approach to same sign top pair production at the LHC and the forward-backward asymmetry at the Tevatron, Phys. Lett. B 703 (2011) 306 [arXiv:1104.1798] [InSPIRE].

[8] C. Degrande, J.M. Gerard, C. Grojean, F. Maltoni and G. Servant, Probing top-Higgs non-standard interactions at the LHC, JHEP 07 (2012) 036 [Erratum ibid. 1303 (2013) 032] [arXiv:1205.1065] [INSPIRE].

[9] M. Fabbrichesi, M. Pinamonti and A. Tonero, Stringent limits on top-quark compositeness from $t \bar{t}$ production at the Tevatron and the LHC, Phys. Rev. D 89 (2014) 074028 [arXiv:1307.5750] [INSPIRE].

[10] M. Fabbrichesi, M. Pinamonti and A. Tonero, Limits on anomalous top quark gauge couplings from Tevatron and LHC data, Eur. Phys. J. C 74 (2014) 3193 [arXiv:1406.5393] [INSPIRE].

[11] Q.-H. Cao, B. Yan, J.-H. Yu and C. Zhang, A general analysis of Wtb anomalous couplings, arXiv: 1504.03785 [INSPIRE].

[12] C. Zhang, Effective field theory approach to top-quark decay at next-to-leading order in QCD, Phys. Rev. D 90 (2014) 014008 [arXiv: 1404.1264] [INSPIRE].

[13] C. Zhang, Effective approach to top-quark decay and FCNC processes at NLO accuracy, J. Phys. Conf. Ser. 556 (2014) 012030 [arXiv:1410.2825] [INSPIRE].

[14] G. Durieux, F. Maltoni and C. Zhang, Global approach to top-quark flavor-changing interactions, Phys. Rev. D 91 (2015) 074017 [arXiv:1412.7166] [INSPIRE].

[15] A. De Rujula, M.B. Gavela, P. Hernández and E. Masso, The selfcouplings of vector bosons: does LEP-1 obviate LEP-2?, Nucl. Phys. B 384 (1992) 3 [InSPIRE].

[16] K. Hagiwara, S. Ishihara, R. Szalapski and D. Zeppenfeld, Low-energy constraints on electroweak three gauge boson couplings, Phys. Lett. B 283 (1992) 353 [INSPIRE].

[17] K. Hagiwara, S. Ishihara, R. Szalapski and D. Zeppenfeld, Low-energy effects of new interactions in the electroweak boson sector, Phys. Rev. D 48 (1993) 2182 [INSPIRE].

[18] S. Alam, S. Dawson and R. Szalapski, Low-energy constraints on new physics revisited, Phys. Rev. D 57 (1998) 1577 [hep-ph/9706542] [INSPIRE].

[19] H. Mebane, N. Greiner, C. Zhang and S. Willenbrock, Constraints on electroweak effective operators at one loop, Phys. Rev. D 88 (2013) 015028 [arXiv:1306.3380] [INSPIRE].

[20] E.E. Jenkins, A.V. Manohar and M. Trott, Renormalization group evolution of the standard model dimension six operators I: formalism and $\lambda$ dependence, JHEP 10 (2013) 087 [arXiv:1308.2627] [INSPIRE].

[21] E.E. Jenkins, A.V. Manohar and M. Trott, Renormalization group evolution of the standard model dimension six operators II: Yukawa dependence, JHEP 01 (2014) 035 [arXiv:1310.4838] [INSPIRE].

[22] R. Alonso, E.E. Jenkins, A.V. Manohar and M. Trott, Renormalization group evolution of the standard model dimension six operators III: gauge coupling dependence and phenomenology, JHEP 04 (2014) 159 [arXiv: 1312.2014] [INSPIRE].

[23] C. Grojean, E.E. Jenkins, A.V. Manohar and M. Trott, Renormalization group scaling of Higgs operators and $\Gamma(h \rightarrow \gamma \gamma)$, JHEP 04 (2013) 016 [arXiv: 1301.2588] [INSPIRE].

[24] J. Elias-Miró, J.R. Espinosa, E. Masso and A. Pomarol, Renormalization of dimension-six operators relevant for the Higgs decays $h \rightarrow \gamma \gamma, \gamma Z$, JHEP 08 (2013) 033 [arXiv:1302.5661] [INSPIRE]. 
[25] J. Elias-Miro, J.R. Espinosa, E. Masso and A. Pomarol, Higgs windows to new physics through $D=6$ operators: constraints and one-loop anomalous dimensions, JHEP 11 (2013) 066 [arXiv:1308.1879] [INSPIRE].

[26] J. Elias-Miró, C. Grojean, R.S. Gupta and D. Marzocca, Scaling and tuning of $E W$ and Higgs observables, JHEP 05 (2014) 019 [arXiv: 1312.2928] [INSPIRE].

[27] B. Grzadkowski, M. Iskrzynski, M. Misiak and J. Rosiek, Dimension-six terms in the standard model lagrangian, JHEP 10 (2010) 085 [arXiv:1008.4884] [INSPIRE].

[28] ATLAS, CMS collaboration, G. Aad et al., Combined measurement of the Higgs boson mass in pp collisions at $\sqrt{s}=7$ and $8 \mathrm{TeV}$ with the ATLAS and CMS experiments, Phys. Rev. Lett. 114 (2015) 191803 [arXiv:1503. 07589] [INSPIRE].

[29] ATLAS, CDF, CMS, D0 collaboration, First combination of Tevatron and LHC measurements of the top-quark mass, arXiv:1403.4427 [INSPIRE].

[30] Particle Data Group collaboration, K.A. Olive et al., Review of particle physics, Chin. Phys. C 38 (2014) 090001 [InSPIRE].

[31] M. Davier, A. Hoecker, B. Malaescu and Z. Zhang, Reevaluation of the hadronic contributions to the muon $g-2$ and to $\alpha_{M Z}$, Eur. Phys. J. C 71 (2011) 1515 [Erratum ibid. C 72 (2012) 1874] [arXiv:1010.4180] [INSPIRE].

[32] CDF, D0 collaboration, T.E.W. Group, 2012 update of the Combination of CDF and DO results for the mass of the $W$ boson, arXiv:1204.0042 [INSPIRE].

[33] Tevatron Electroweak Working Group, CDF, DelPhi, SLD Electroweak and Heavy Flavour Groups, AlePh, LEP Electroweak Working Group, SLD, OPAL, D0, L3 collaboration, L.E.W. Group, Precision electroweak measurements and constraints on the standard model, arXiv:1012.2367 [INSPIRE].

[34] Sld Electroweak Group, Delphi, Aleph, SLD, Sld Heavy Flavour Group, OPAL, LEP Electroweak Working Group, L3 collaboration, S. Schael et al., Precision electroweak measurements on the $Z$ resonance, Phys. Rept. 427 (2006) 257 [hep-ex/0509008] [INSPIRE].

[35] M. Awramik, M. Czakon, A. Freitas and G. Weiglein, Precise prediction for the $W$ boson mass in the standard model, Phys. Rev. D 69 (2004) 053006 [hep-ph/0311148] [INSPIRE].

[36] M. Awramik, M. Czakon and A. Freitas, Electroweak two-loop corrections to the effective weak mixing angle, JHEP 11 (2006) 048 [hep-ph/0608099] [INSPIRE].

[37] M. Awramik, M. Czakon, A. Freitas and B.A. Kniehl, Two-loop electroweak fermionic corrections to $\sin ^{2} \theta^{b} \bar{b}_{\text {eff }}$, Nucl. Phys. B 813 (2009) 174 [arXiv:0811.1364] [INSPIRE].

[38] A. Freitas, Higher-order electroweak corrections to the partial widths and branching ratios of the $Z$ boson, JHEP 04 (2014) 070 [arXiv: 1401.2447] [INSPIRE].

[39] L. Berthier and M. Trott, Towards consistent electroweak precision data constraints in the SMEFT, JHEP 05 (2015) 024 [arXiv: 1502.02570] [INSPIRE].

[40] Z. Han and W. Skiba, Effective theory analysis of precision electroweak data, Phys. Rev. D 71 (2005) 075009 [hep-ph/0412166] [INSPIRE].

[41] F. del Aguila and J. de Blas, Electroweak constraints on new physics, Fortsch. Phys. 59 (2011) 1036 [arXiv:1105.6103] [INSPIRE].

[42] M. Ciuchini, E. Franco, S. Mishima and L. Silvestrini, Electroweak precision observables, new physics and the nature of a $126 \mathrm{GeV}$ Higgs boson, JHEP 08 (2013) 106 [arXiv:1306.4644] [INSPIRE]. 
[43] J. de Blas, Electroweak limits on physics beyond the standard model, EPJ Web Conf. 60 (2013) 19008 [arXiv: 1307.6173] [INSPIRE].

[44] A. Pomarol and F. Riva, Towards the ultimate SM fit to close in on Higgs physics, JHEP 01 (2014) 151 [arXiv: 1308.2803] [INSPIRE].

[45] J. Ellis, V. Sanz and T. You, The effective standard model after LHC Run I, JHEP 03 (2015) 157 [arXiv: 1410.7703] [INSPIRE].

[46] A. Efrati, A. Falkowski and Y. Soreq, Electroweak constraints on flavorful effective theories, JHEP 07 (2015) 018 [arXiv:1503.07872] [INSPIRE].

[47] J. de Blas, M. Chala, M. Pérez-Victoria and J. Santiago, Observable effects of general new scalar particles, JHEP 04 (2015) 078 [arXiv:1412.8480] [INSPIRE].

[48] J. de Blas et al., in preparation.

[49] J. de Blas et al., Global bayesian analysis of the Higgs-boson couplings, arXiv:1410.4204 [INSPIRE].

[50] C. Zhang, N. Greiner and S. Willenbrock, Constraints on non-standard top quark couplings, Phys. Rev. D 86 (2012) 014024 [arXiv:1201.6670] [InSPIRE].

[51] C. Hartmann and M. Trott, On one-loop corrections in the standard model effective field theory; the $\Gamma(h \rightarrow \gamma \gamma)$ case, JHEP 07 (2015) 151 [arXiv: 1505.02646] [INSPIRE].

[52] M. Ghezzi, R. Gomez-Ambrosio, G. Passarino and S. Uccirati, NLO Higgs effective field theory and $\kappa$-framework, JHEP 07 (2015) 175 [arXiv:1505.03706] [INSPIRE].

[53] M. Carpentier and S. Davidson, Constraints on two-lepton, two quark operators, Eur. Phys. J. C 70 (2010) 1071 [arXiv: 1008. 0280] [INSPIRE].

[54] M.E. Peskin and T. Takeuchi, Estimation of oblique electroweak corrections, Phys. Rev. D 46 (1992) 381 [INSPIRE].

[55] A. Pomarol and J. Serra, Top quark compositeness: feasibility and implications, Phys. Rev. D 78 (2008) 074026 [arXiv:0806.3247] [InSPIRE].

[56] C. Anastasiou, E. Furlan and J. Santiago, Realistic composite Higgs models, Phys. Rev. D 79 (2009) 075003 [arXiv:0901.2117] [inSPIRE].

[57] F. del Aguila, M. Pérez-Victoria and J. Santiago, Observable contributions of new exotic quarks to quark mixing, JHEP 09 (2000) 011 [hep-ph/0007316] [INSPIRE].

[58] F. del Aguila, J. de Blas and M. Pérez-Victoria, Electroweak limits on general new vector bosons, JHEP 09 (2010) 033 [arXiv: 1005.3998] [INSPIRE].

[59] B. Grzadkowski, Z. Hioki and M. Szafranski, Four Fermi effective operators in top quark production and decay, Phys. Rev. D 58 (1998) 035002 [hep-ph/9712357] [INSPIRE].

[60] H. Baer et al., The International Linear Collider technical design report - Volume 2: physics, arXiv:1306.6352 [INSPIRE].

[61] TLeP Design Study Working Group collaboration, M. Bicer et al., First look at the physics case of TLEP, JHEP 01 (2014) 164 [arXiv:1308.6176] [INSPIRE].

[62] E. Devetak, A. Nomerotski and M. Peskin, Top quark anomalous couplings at the International Linear Collider, Phys. Rev. D 84 (2011) 034029 [arXiv:1005.1756] [InSPIRE].

[63] ATLAS collaboration, Search for production of vector-like quark pairs and of four top quarks in the lepton-plus-jets final state in pp collisions at $\sqrt{s}=8 \mathrm{TeV}$ with the ATLAS detector, JHEP 08 (2015) 105 [arXiv: 1505.04306] [INSPIRE].

[64] A. Buckley et al., A global fit of top quark effective theory to data, arXiv:1506.08845 [INSPIRE]. 